Torreón Universitario

www.faremcarazo.unan.edu.ni / ISSN 2410-5708 / e-ISSN 2313-7215

Año 8 | Núm. 23 | pág. 58 - pág. 72 | octubre 2019 - enero 2020
Esta obra está bajo una licencia de Creative Commons

Atribución-NoComercial-SinDerivar 4.0 Internacional

\title{
Contaminación natural por arsénico en las aguas subterráneas de la comunidad rural "La Fuente", para sugerir y promover el uso de fuentes alternativas de agua segura municipio La Paz Centro, León, Nicaragua
}

MSc. Maximina Altamirano Laboratorio de Contaminantes metálicos. Centro para la

Investigación en Recursos Acuáticos de Nicaragua UNAN-Managua maximina.altamirano@cira.unan.edu.ni

MSc. Valeria Delgado Área de Docencia. Centro para la Investigación en Recursos Acuáticos de Nicaragua UNAN-Managua valeria.delgado@cira.unan.edu.ni

Palabras clave: contaminación, alteración hidrotermal, arsénico, fuentes geogénicas

\section{RESUMEN} studios previos han revelado que en Nicaragua la presencia de arsénico es de origen geogénico por lo que es común encontrarlo en aguas tanto superficiales como subterráneas, suelo, rocas y sedimentos; además de encontrarse en los complejos volcánicos, fumarolas, fuentes termales, gases y fluidos geotérmicos. Fue hasta el 2010 cuando el arsénico fue detectado en materiales volcánicos y sedimentarios del Cuaternario presentes en el arco volcánico de la Cordillera de Los Maribios. Es así, que en la Comunidad de La Fuente 
localizada al NE del complejo volcánico Momotombo-El Hoyo y cerca de la Caldera Monte Galán, donde 112 familias se abastecen de agua subterránea, se determinó la concentración de arsénico total en agua de 30 pozos excavados, tres pozos perforados y un manantial. Las concentraciones de arsénico oscilaron en el rango desde $2 \mu \mathrm{g} \cdot \mathrm{l}^{-1}$ (Casas de Rafaela Rojas y Porfirio Reyes) hasta $103 \mu \mathrm{g} \cdot \mathrm{l}^{-1}$ en el manantial Aguas Calientes (La Chistatosa). El 70\% de las muestras analizadas (24 fuentes) exceden el límite nacional adoptado por el país de $10 \mu \mathrm{g} \mathrm{As} \cdot \mathrm{l}^{-1}$ para agua de consumo humano. Los sitios con las mayores temperaturas $\left(35,6\right.$ a $\left.45,8{ }^{\circ} \mathrm{C}\right)$ son los que presentan las mayores concentraciones de arsénico (38 a $103 \mu \mathrm{g} \mathrm{l}^{-1}$ ), mayores valores de conductividad eléctrica $\left(983-1140 \mu \mathrm{S} \cdot \mathrm{cm}^{-1}\right)$ y menores valores de $\mathrm{pH}(5,80-7,08$ unidades). Los pobladores fueron informados sobre el riesgo al que están expuestos por ingerir agua contaminada con altos valores de este tóxico; a la vez que se identificaron fuentes seguras de agua para consumo humano, las cuales fueron recomendadas a los usuarios. Esta investigación ${ }^{1}$, además de generar información científica, se convierte en una herramienta para apoyar en la toma de decisiones por parte de las autoridades, y cambio por parte de los pobladores locales en el uso de fuentes alternativas para agua de consumo humano y de preparación de sus alimentos.

\section{INTRODUCCIÓN}

El arsénico es el metaloide con el más alto potencial de toxicidad para la salud humana que existe en la naturaleza, encontrándose generalmente en concentraciones traza y cuya movilidad y por ende toxicidad dependen de procesos bióticos y abióticos (WHO, 2011). La contaminación por arsénico es uno de los grandes problemas ambientales a nivel mundial en países como Argentina, Chile, Brasil, Perú, Bolivia, México, Tailandia, Bangladesh, China, India y Estados Unidos, incluyendo a Nicaragua (Anawar, et al., 2003).

En Nicaragua, se han reportado casos de arsenicosis (envenenamiento por arsénico), tal fue el caso de la comunidad de El Zapote, Valle de Sébaco (Gómez C., 2002) donde más de 70 personas consumieron agua con $1320 \mu \mathrm{g} / \mathrm{L}$ de arsénico durante año y medio; presentando toxicidad aguda y crónica [(Gómez C., 2002), (Gómez Cuevas, 2007)]; por lo cual en Nicaragua existe una preocupación ambiental y de salud pública al detectarse altos niveles de arsénico en el agua subterránea. Se ha encontrado arsénico en aguas subterráneas en las regiones noroeste y suroeste del país, próximas a áreas mineralizadas a lo largo de la estructura tectónica activa más importante, la Depresión o Graben de Nicaragua y en la Plataforma Central (Altamirano, 2005), así como también en zonas aledañas a la Cordillera volcánica de Los Maribios [(Nuevas Esperanzas \& Longley, 2010), (ORGANIZACIÓN PANAMERICANA DE LA SALUD, OPS/OMS Nicaragua, \& Nuevas Esperanzas, 2011), (Nuevas Esperanzas, 2013)] y que son la única fuente de agua disponible para la población rural en muchas comunidades del país, como las ubicadas

1. Las autoras agradecen a los Fondos para Proyectos de Investigación FPI 2025-2017 de la UNAN-Managua por medio del proyecto No. 12201504 por el apoyo económico otorgado para la ejecución de esta investigación. 
al norte del municipio de La Paz Centro, que es este caso de estudio. La presencia de arsénico en estas comunidades es atribuida a un intenso estrés tectónico y a la presencia de diferentes sistemas de fallas activas y no activas, fracturas y alteración hidrotermal a lo largo de fallas.

El área de estudio se ubica en un ambiente tectónico activo y posee una compleja geología con rocas ígneas y sedimentarias del Cuaternario aluvial y volcánico; factores que predisponen a la contaminación del medio ambiente natural y de las aguas subterráneas, como es el caso del arsénico; esto es uno de los principales contaminantes presentes de manera natural en las matrices ambientales y que es causante de efectos adversos en la salud por la ingestión de aguas contaminadas con este metaloide (Armienta \& Segovia, 2008).

El agua es la principal vía de entrada en la cadena alimentaria humana a través de la ingesta y el consumo de alimentos de origen vegetal (arroz, verduras, hortalizas, cereales) y animal (carnes, pescado, leche) que han sido producidos con agua contaminada con este genotóxico (ATSDR, 2007). Las manifestaciones clínicas de la intoxicación aguda y crónica por arsénico están asociadas con diversas formas de enfermedades de la piel y daños en órganos internos, alteraciones cardiovasculares, renales, circulatorias y respiratorias y por último cáncer (Hossain, 2006).

Debido a este alto potencial como agente carcinógeno es que se han establecido normas o valores guía que regulen la presencia de arsénico en las aguas subterráneas las que varían según los países. Nicaragua a través del Instituto Nicaragüense de Acueductos y Alcantarillados (INAA, 2001), adoptó los valores guías establecidos por la Organización Mundial de la Salud (OMS), la cual establece como norma de calidad para agua de consumo humano $10 \mu \mathrm{g} \cdot \mathrm{l}^{-1}$ de arsénico (OMS, 2011).

\section{Área de estudio}

El área de estudio es la comunidad rural de La Fuente, ubicada en la base de las faldas septentrionales del complejo volcánico Momotombo-El Hoyo y del edificio de la caldera Monte Galán; dentro de la provincia geomorfológica de la Depresión Nicaragüense. Tiene una extensión aproximada de $15 \mathrm{~km}^{2}$ y se localiza en la parte noreste del municipio de La Paz Centro, Departamento de León (figura 1). La comunidad está conformada por 1,300 habitantes distribuidos en 121 familias, quienes cuentan con una red de distribución de agua potable a través de un pozo perforado comunitario; el resto de las familias se suministran a través de pozos excavados (86) y de dos pozos perforados familiares. La comunidad no cuenta con sistema de alcantarillado, por lo que cada casa cuenta con letrina o fosa séptica. 


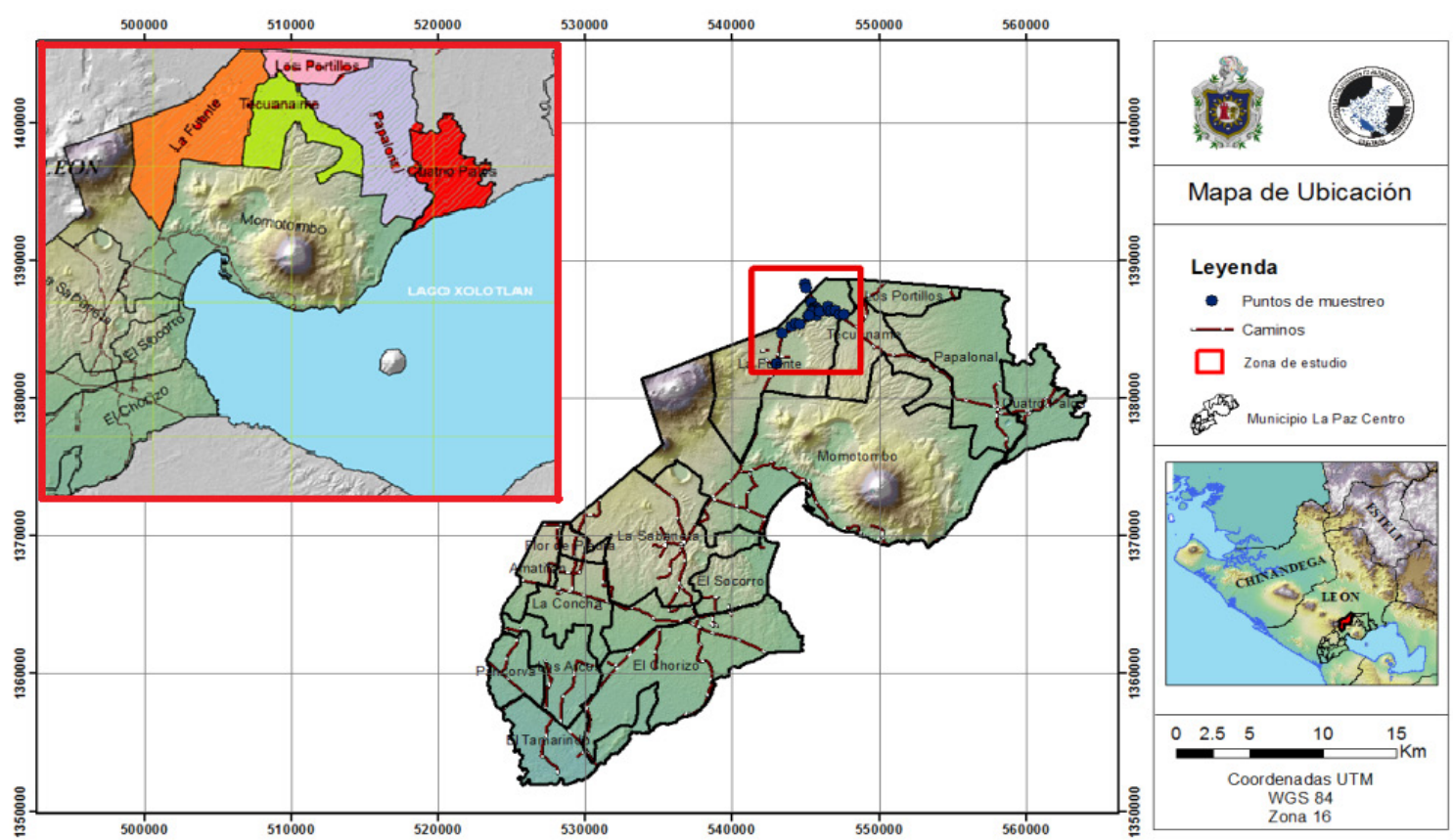

Figura 1. Macro y micro localización de la comunidad rural La Fuente, La Paz Centro.

\section{METODOLOGÍA}

\section{Materiales y métodos}

Para llevar a cabo el presente estudio, inicialmente se realizó una gira de reconocimiento por las comunidades establecidas en las faldas del complejo volcánico Momotombo-El Hoyo, reuniéndose el personal ejecutor del proyecto con los líderes comunitarios de las comunidades de Cuatro Palos, El Papalonal, Tecuaname, Los Portillos y La Fuente; con el fin de conocer la problemática relacionada con el agua de consumo humano y las posibles afectaciones a la salud a través de este recurso.

Una vez obtenida la información de los comunitarios, se procedió a realizar una compilación detallada de estudios geológicos, químicos e hidrogeológicos disponibles en el área con el fin de tener una caracterización general del entorno físico y geológico de la zona, así como de la química de las aguas subterráneas de la región y también sobre los estudios previos relacionados con la salud poblacional en ese sector, ya que la mayor prevalencia de enfermedades presentadas por los pobladores de La Fuente es la insuficiencia renal crónica.

Se identificaron 90 objetos hidrogeológicos (pozos excavados, pozos perforados y manantiales) de los cuales se muestrearon 34 sitios para determinar las concentraciones de arsénico total, considerando aquellos pozos en los que un mayor número poblacional hiciera uso de ellos, así como también en aquellas familias que presentan afectaciones en los riñones. Estos puntos se distribuyeron de la siguiente manera: 30 pozos excavados (PE), 3 pozos perforados (PP) y un manantial (Mn). 

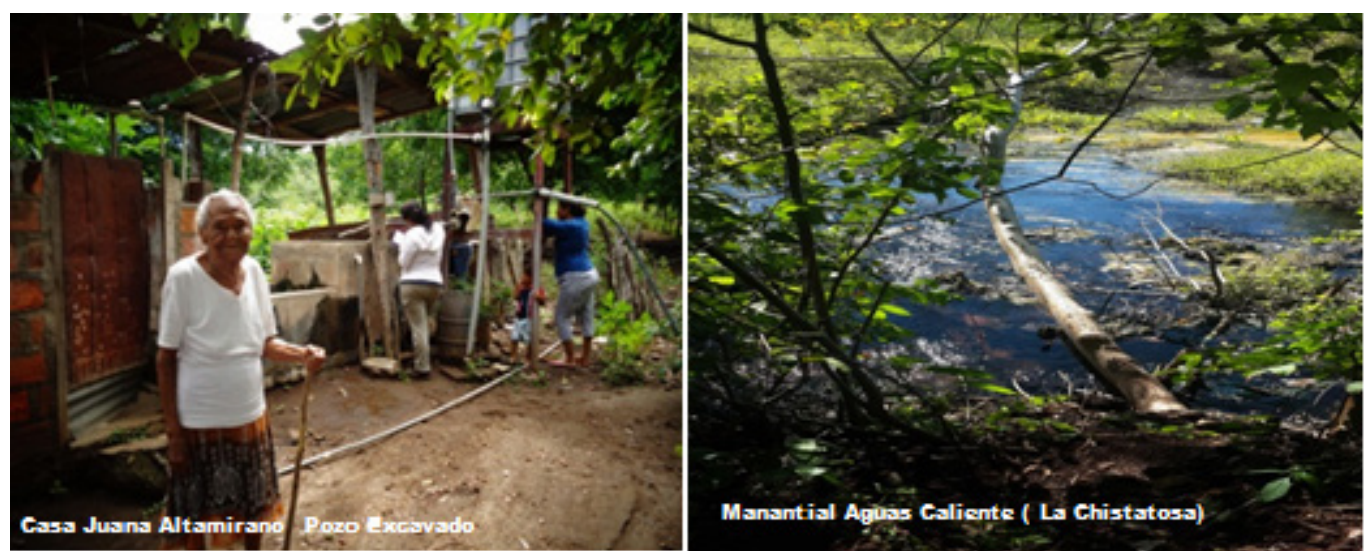

Figura 2. Puntos muestreados: pozo excavado Casa de Juana Altamirano (A) y manantial Aguas Calientes conocido como La Chistatosa (B), de la comunidad rural La Fuente.

Todos los puntos muestreados fueron geo-referenciados empleando un Sistema de Posicionamiento Global (GPS); a la vez que a cada punto de muestreo se le realizaron mediciones de parámetros físicos y químicos in situ, como el $\mathrm{pH}$, potencial redox (Eh), temperatura (T) y conductividad eléctrica ( $\mathrm{CE}$ ); utilizando equipos de medición portátil. La metodología de toma de muestra empleada fue la establecida en el manual de procedimientos del Laboratorio de Contaminantes Metálicos del CIRA/UNAN-Managua (PROC-CM-02), para la colecta de muestras de agua para análisis de metales pesados.

El arsénico total en las muestras de agua se determinó usando la técnica de generación de hidruros por medio de un espectrofotómetro de Absorción Atómica Varian SpectrAA240FS acoplado a un VGA 77. La metodología de digestión y análisis es la establecida en el Standard Methods for the examination of Water and Wastewater (SMWW, 2012; 22nd edition), siguiendo las recomendaciones y procedimientos de los métodos 3114B (3114B, Espectroscopía de absorción atómica), método continúo de generación de hidruros (VGA) y 3030F (Digestión con Ácido Clorhídrico); respectivamente. El límite de detección del método es de 0,99 $\mu \mathrm{g}^{-\mathrm{l}^{-1} \mathrm{de}}$ arsénico total en agua. Cada muestra fue analizada por duplicado, y con cada lote de muestras se analizaron muestras controles, muestras certificadas, así como los porcentajes de recuperación de las muestras, los que estuvieron en el rango de 94 y $96 \%$.

\section{RESULTADOS}

En la Tabla 1 se muestran los valores obtenidos para los parámetros de campo y la concentración de As total encontrados en los sitios monitoreados en la comunidad de La Fuente. 
Tabla 1. Parámetros físico-químicos de campo y contenido de arsénico total en aguas subterráneas de la comunidad rural La Fuente, La Paz Centro, León - junio 2016.

\begin{tabular}{|c|c|c|c|c|c|}
\hline Puntos muestreados & $\begin{array}{c}\mathrm{pH} \\
\text { (Und) }\end{array}$ & $\begin{array}{l}\text { Temp } \\
\left({ }^{\circ} \mathrm{C}\right)\end{array}$ & $\begin{array}{c}\text { CE } \\
\left(\mu \mathrm{S} \cdot \mathrm{cm}^{-1}\right)\end{array}$ & $\begin{array}{c}\text { Pot. } \\
\text { Redox } \\
(\mathbf{m V})\end{array}$ & $\begin{array}{l}\text { As total } \\
\left(\mu \mathrm{g} \cdot \mathrm{l}^{-1}\right)\end{array}$ \\
\hline $\begin{array}{l}\text { 1-Manantial Aguas Calientes } \\
\text { (La Chistatosa) }\end{array}$ & 5,80 & 45.8 & 1140 & 168,5 & 103,00 \\
\hline 2-PE Porfirio Altamirano & 6,88 & 35,1 & 1119 & 295,8 & 34,84 \\
\hline 3-Pozo El Tránsito & 7,08 & 35,6 & 983 & 314,5 & 55,70 \\
\hline 4-PE -Santa Rosa & 7,01 & 36,6 & 1132 & 194,5 & 50,90 \\
\hline 5-PE Manuel Araúz & 6,69 & 32,9 & 995 & 298,5 & 22,74 \\
\hline 6-PE Adelaida del Socorro & 6,76 & 30,6 & 914 & 325,2 & 21,05 \\
\hline 7-PE Narciso Araúz & 6,77 & 31,0 & 916 & 320,8 & 20,94 \\
\hline 8-PE Franklin Araúz P & 6,51 & 30,0 & 910 & 391,6 & 19,44 \\
\hline 9-Finca Reyes Altamirano & 6,70 & 31,4 & 945 & 366,9 & 23,32 \\
\hline 10-PE Juana Altamirano & 6,86 & 34,6 & 945 & 350,2 & 15,99 \\
\hline 11-PE Arturo Altamirano & 6,94 & 32,5 & 961 & 368,6 & 20,38 \\
\hline 12-PE Rubén Hernández & 6,77 & 31,5 & 915 & 347,6 & 11,32 \\
\hline 13-PP Comunidad La Fuente & 7,06 & 35,7 & 925 & 330,7 & 12,86 \\
\hline 14-PE Arsenio Guido & 6,84 & 32,7 & 914 & 351,3 & 12,26 \\
\hline 15-PE Dora Ramírez & 7,48 & 35,1 & 905 & 278,5 & 10,9 \\
\hline 16-PE Pedro Castillo & 6,65 & 31,1 & 791 & 165,1 & 23,17 \\
\hline 17-PE Isabel Rojas & 6,54 & 31,0 & 532 & 200,8 & 23,52 \\
\hline 18-PE Socorro Marenco & 6,43 & 31,8 & 761 & 209,2 & 21,58 \\
\hline 19-PE Concepción Vargas & 6,59 & 29,3 & 799 & 226,0 & 15,41 \\
\hline 20-PE Esperanza Vargas & 6,52 & 31,6 & 741 & 245,6 & 14,8 \\
\hline 21-PE Dominga Rojas & 6,74 & 31,9 & 818 & 215,4 & 10,41 \\
\hline 22-PE Zelma Reyes & 6,51 & 30,9 & 784 & 221,4 & 12,75 \\
\hline 23-PE Yelba Hernández & 7,61 & 32,3 & 594 & 161,3 & 10,19 \\
\hline 24-PP Gertrudis Treminio & 6,82 & 32,7 & 963 & 291,2 & 17,37 \\
\hline 25-PE Gertrudis Treminio & 6,70 & 31,2 & 844 & 241,5 & 9,09 \\
\hline
\end{tabular}




\begin{tabular}{|c|c|c|c|c|c|}
\hline Puntos muestreados & $\begin{array}{c}\text { pH } \\
\text { (Und) }\end{array}$ & $\begin{array}{c}\text { Temp } \\
\left({ }^{\circ} \mathrm{C}\right)\end{array}$ & $\begin{array}{c}C E \\
\left(\mu S \cdot \mathrm{cm}^{-1}\right)\end{array}$ & $\begin{array}{c}\text { Pot. } \\
\text { Redox } \\
\text { (mV) }\end{array}$ & $\begin{array}{c}\text { As total } \\
\left(\boldsymbol{\mu g} \cdot \mathbf{1}^{-1}\right)\end{array}$ \\
\hline \multicolumn{6}{|l|}{ « tabla viene de página anterior } \\
\hline 26-PE Wilber Espinoza & 6,36 & 31,6 & 512 & 227,3 & 9,20 \\
\hline 27-PE Marvin Moya & 6,70 & 33,4 & 813 & 243,1 & 5,86 \\
\hline 28-PE Pedro Vill Mejía & 6,68 & 31,7 & 547 & 246,9 & 4,42 \\
\hline 29-PE Hermógenes A & 7,02 & 31,9 & 745 & 238,2 & 6,91 \\
\hline 30-PE Martin Espinoza Rojas & 7,43 & 32,0 & 525 & 232,4 & 7,04 \\
\hline 31-PE Lucía Castillo & 6,98 & 31,0 & 476 & 263,6 & 5,30 \\
\hline 32-PE Rafaela Rojas & 6,11 & 30,7 & 211 & 433,1 & 2,19 \\
\hline 33-PE Porfirio Mayorga & 6,70 & 31,0 & 514 & 374,7 & 2,21 \\
\hline 34-PE Leonila Altamirano E & 6,63 & 32,4 & 256 & 341,1 & $<0,99$ \\
\hline Mínimo & 5,80 & 29,3 & 211 & 161,3 & $<0,99$ \\
\hline Máximo & 7,61 & 36,6 & 1140 & 433,1 & 103,00 \\
\hline $\begin{array}{l}\text { PE: Pozo excavado } \\
\text { PP: Pozo perforado }\end{array}$ & \multicolumn{3}{|c|}{ CE: conductividad eléctrica } & & \\
\hline
\end{tabular}

\section{Parámetros de campo (pH, Temperatura, Conductividad eléctrica y Potencial REDOX)}

La medición de estos parámetros in situ, proporciona información relevante sobre las condiciones de un acuífero en un momento e instante en particular con respecto a la acidez o basicidad de las aguas, las condiciones de temperatura (posibles fuentes de termalismo), los ambientes oxidantes o reductores que conllevan a la liberación de especies más tóxicas como es el caso del arsénico, por mencionar algunos.

La conductividad eléctrica en el $94 \%$ de los puntos muestreados supera el valor recomendado por las normas (CAPRE, 1994) para consumo humano el cual es de $400 \mu \mathrm{S} \cdot \mathrm{cm}^{-1}$. Sin embargo, los valores reportados de conductividad eléctrica en los pozos monitoreados, afectan la calidad organoléptica del agua, específicamente en el sabor de ésta, disminuyendo su aceptación por parte de los consumidores (OMS, 2006).

La temperatura es uno de los parámetros más importantes de la calidad del agua y afecta la química de esta ya que regula parámetros como el $\mathrm{pH}$, oxígeno disuelto, potencial REDOX y las concentraciones iónicas presentes en el agua. La temperatura del agua subterránea en un punto y momento determinado representa un estado de equilibrio entre los "aportes" y las “extracciones". Se determinó que los pozos con mayor temperatura (tabla 1) también reportaron 
las mayores concentraciones de arsénico; tal es el caso del manantial Aguas Calientes con la mayor temperatura $\left(45,8^{\circ} \mathrm{C}\right)$.

Las mediciones de $\mathrm{pH}$ se ejecutan en una escala de 0 a 14, con 7.0 considerado neutro. Las soluciones con un $\mathrm{pH}$ inferior a 7 unidades de $\mathrm{pH}$ se consideran ácidas; mientras que las que presentan un $\mathrm{pH}$ superior a 8 unidades de $\mathrm{pH}$ son consideradas alcalinas. De los pozos muestreados un 76,5 \% (27 pozos) obtuvieron valores con $\mathrm{pH}$ inferior a 7 , siendo considerados de ligeramente ácidos a ácidos, mientras que el 20.5\% (7 pozos) presentan valores que superan levemente el pH neutro, considerándose ligeramente alcalinos. El valor más bajo de pH (5,80 unidades) se encontró en el manantial Aguas Caliente (La Chistatosa), que a su vez reportó la mayor temperatura y concentración de arsénico total. El estado de oxidación del arsénico, y por tanto su movilidad, se encuentran controlado fundamentalmente por las condiciones REDOX (potencial REDOX, Eh) y el pH.

Los valores de potencial REDOX en el área de estudio oscilan entre 168,5 y 433,10 mV. Al relacionar el potencial REDOX con los valores de arsénico total de la comunidad rural La Fuente, no se encontró una correlación significativa con todos los puntos analizados, pero sí con el manantial Aguas Caliente (La Chistatosa). En este punto se reportó la mayor concentración de arsénico y de los menores valores de Eh indicando condiciones reductoras, probablemente esto se deba a que este sitio está siendo controlado por distintos procesos de oxidación-reducción debido al desgaste de los minerales químicos y las condiciones REDOX cambiantes las cuales aumentan la movilidad del arsénico y facilitan la transferencia desde la forma sólida a la disuelta (Smedley y Kinniburgh, 2002).

\section{Arsénico Total en Aguas subterráneas}

Durante el reconocimiento geológico e hidrogeológico, se observó que es común encontrar ambos lados de la carretera, material (rocas) alterado hidrotermalmente (figura 3); a la vez que este tipo de rocas están presentes tanto en los pozos excavados como en los cortes de carretera.

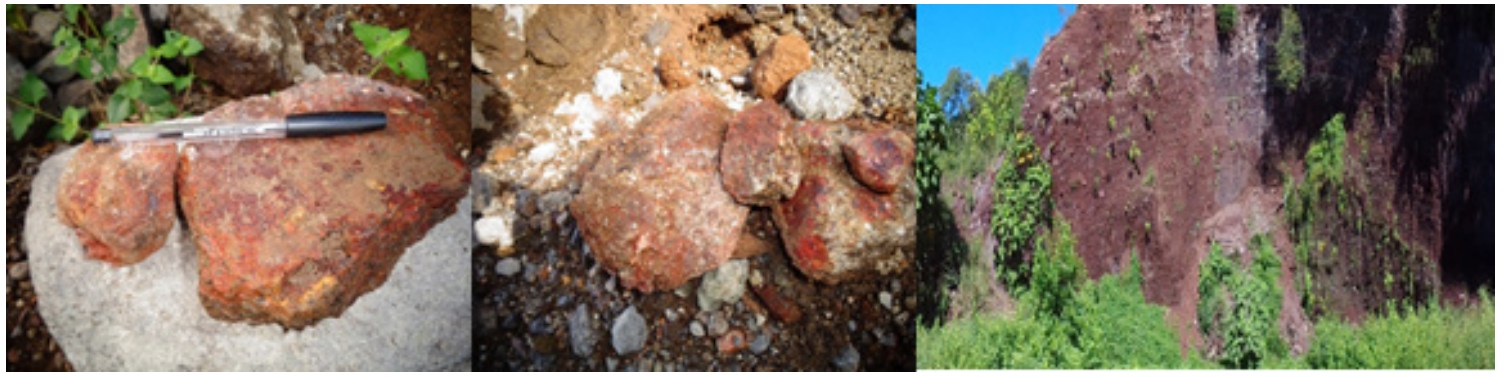

Figura 3. Rocas alteradas en comunidad rural La Fuente, frente a casa de Narciso Arauz.

El $70 \%$ de los puntos muestreados reportan concentraciones de arsénico total que superan el valor guía de $10 \mu \mathrm{g} \cdot \mathrm{l}^{-1}$ de arsénico en agua establecido para consumo humano (Figura 4), lo cual es un peligro latente para la salud de la población de La Fuente. 


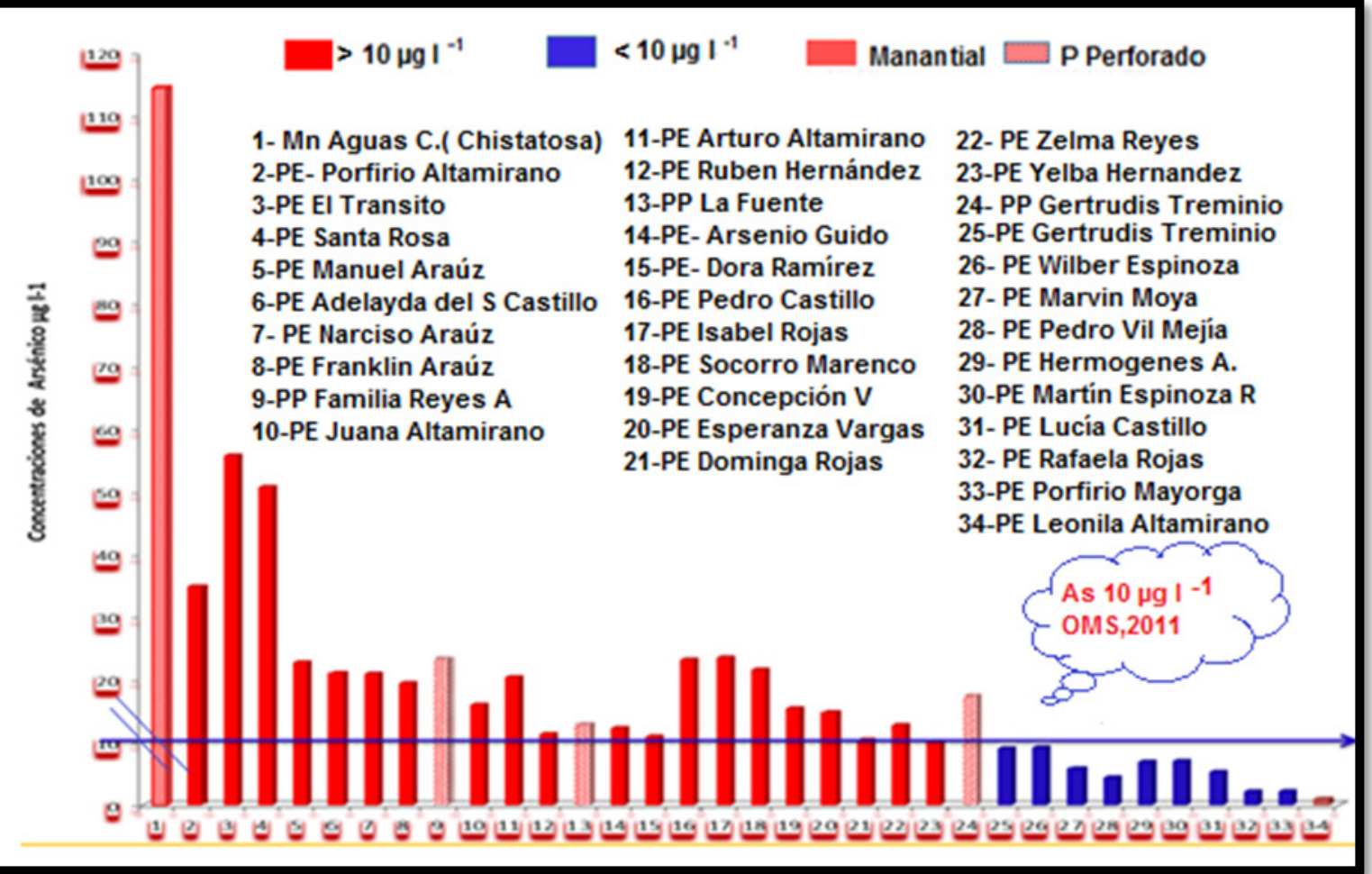

Figura 4. Concentraciones de arsénico detectadas en pozos excavados y perforados y un manantial en la comunidad rural "La Fuente".

Cabe mencionar que los pobladores profundizan anualmente los pozos debido a que en el verano estos se secan. Estas acciones generan el riesgo de incrementar las concentraciones del tóxico en el agua de consumo, representando esto un factor condicionante para el aprovechamiento del agua subterránea profunda proveniente de medios alterados (Kinniburgh, D G; Smedley, P L; Summary, 2001).

El manantial Aguas Calientes es una fuente termal que nace del Cerro La Chistatosa (figura 5); se ubica en la parte norte de puerto Momotombo, es de poco caudal y recorre el municipio de norte a sur. En esta fuente de agua, se detectó el valor más alto de arsénico (103 $\left.\mu \mathrm{g} \cdot \mathrm{l}^{-1}\right)$, concentración que es similar a la reportada en los termales de Tipitapa (102 $\mu \mathrm{g} \cdot \mathrm{l}^{-1}$; (CIRA/ UNAN-Managua \& Laboratorio Contaminantes Metálicos, 2009). El agua de esta manantial descarga en el Lago Xolotlán, presentando un fuerte olor a sulfuro y temperatura cercana a los $50{ }^{\circ} \mathrm{C}$. El área en donde se localiza este manantial muestra una intensa alteración hidrotermal lo que pudiera provocar la disolución de las rocas que son la fuente principal del arsénico en las aguas subterráneas. 


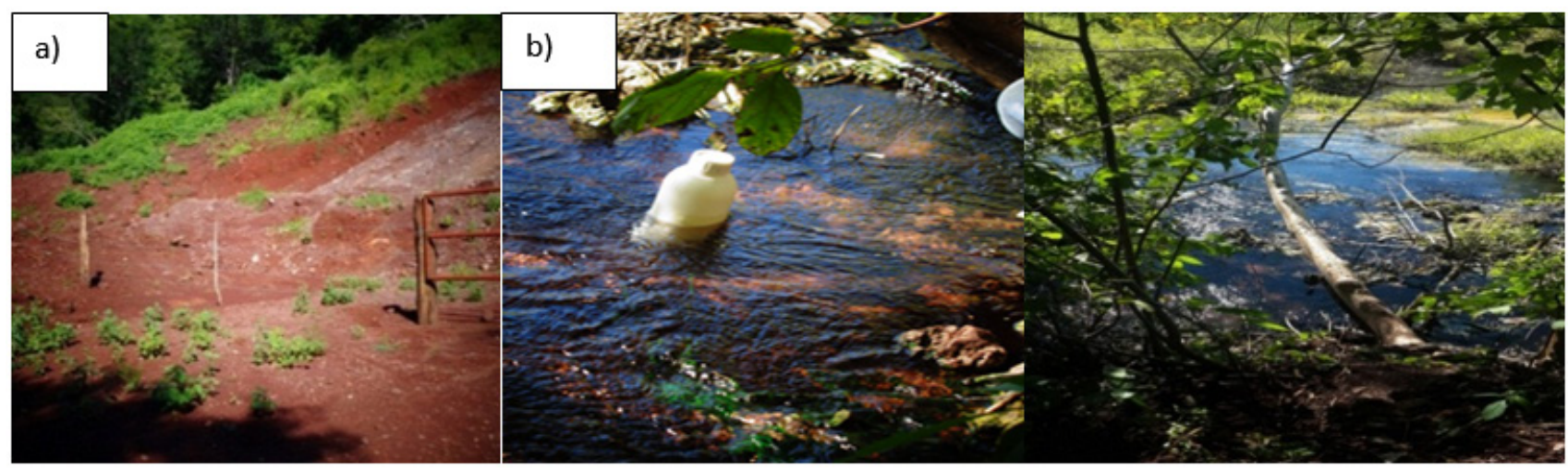

Figura 5. a) Cerro La Chistatosa y b) manantial de aguas termales conocido como Aguas Caliente que surge de este cerro.

De acuerdo con (Boyle R, 1979), la intensa alteración hidrotermal vuelve vulnerable a la roca por no tener capacidad de mantener el $\mathrm{pH}$ constante, incrementando la acidez y disminuyendo la capacidad de neutralizar las reacciones químicas, y de esta manera facilitando la incorporación de muchos metales al manto acuífero por percolación de elementos nocivos como el arsénico a las aguas subterráneas y superficiales.

Tal como se mencionó anteriormente, los puntos que presentan las mayores temperaturas, menor $\mathrm{pH}$ y conductividades eléctricas altas, son los que presentaron las mayores concentraciones de arsénico (Manantial Aguas Caliente, PE Porfirio Altamirano, PE Santa Rosa, PE El Tránsito, PE Manuel Arauz, PE Adelaida del Socorro Castillo, PE Narciso Arauz, PE Franklin Arauz P. y PP Finca Reyes Altamirano). Estos sitios están ubicados sobre una antigua caldera volcánica conocida como Galán causada por el hundimiento de una cámara magmática y que actualmente forman parte de extensas planicies (OLADE; 1982). Estas concentraciones naturales de arsénico podrían estar relacionadas al proceso de contaminación de acuíferos someros por parte de fluidos geotérmicos los cuales también inciden en el aumento de temperatura en estos sitios (Electroconsult, 2001).

En el área del centro de la comunidad, las concentraciones de arsénico si bien fueron menores a las mencionadas anteriormente, siguen superando el valor guía adoptado por el país con concentraciones entre el 20,38 $\mu \mathrm{g} \cdot \mathrm{l}^{-1}$ y $10,90 \mu \mathrm{g} \cdot \mathrm{l}^{-1}$; cabe destacar que estos pozos tienen una profundidad entre 20 y 25 metros. Antes del 2013, el PP Comunidad La Fuente, abastecía únicamente a 7 familias, con una concentración de arsénico de 17,00 $\mu \mathrm{g} \cdot \mathrm{l}^{-1}$; para el 2013 el INAA seleccionó este pozo para uso comunitario, abasteciendo a un total de 112 familias. En el presente estudio, este pozo fue monitoreado reportando una concentración de $12,86 \mu \mathrm{g} \cdot \mathrm{l}^{-1}$, por lo que se observa una disminución en el arsénico presente en el agua; esto se debe probablemente a que a mayor extracción de agua en el pozo, el nivel freático busca una nueva cota de equilibrio para estabilizarse provocando mezclas de aguas de diferentes calidades donde puede ser afectadas las concentración del contaminante (Smedley, Nicolli, Macdonald, \& Barros, 2002). 


\section{Identificación de fuentes seguras}

En la actualidad no existe una cura eficaz para la intoxicación crónica con arsénico; sin embargo, en los casos de intoxicación aguda es posible revertir los efectos nocivos para la salud de las personas mientras se encuentren en las primeras tres etapas (pre-clínica, clínica y complicación) si se reemplaza la fuente de agua de bebida por una libre de arsénico ya sea que cumpla con el valor establecido por la (OMS, 2011) de $10 \mu \mathrm{g} \cdot \mathrm{l}^{-1}$, o con el uso de técnicas de remediación es la única medida para contrarrestar la prevalencia del tóxico en el ser humano y se puede considerar que su recuperación es casi completa.

En el presente estudio se identificaron aquellos pozos que presentaron las menores concentraciones de arsénico, siendo estos los sitios: PE Rafaela Rojas (2,19 $\left.\mu \mathrm{g} \cdot \mathrm{l}^{-1}\right)$ y PE Porfirio Mayorga $\left(2,21 \mu \mathrm{g} \cdot \mathrm{l}^{-1}\right)$; los cuales fueron recomendados a los pobladores de La Fuente. En cuanto al pozo comunitario de distribución que poseen actualmente PP Comarca La Fuente, se recomienda que podría ser usado para usos secundarios (lavar ropa, regar plantas, bañarse, breva de ganado y cultivos). Sin embargo, cabe mencionar, que las sustancias que producen cáncer (carcinógenos), como el arsénico, siempre representan riesgo a cualquier nivel.

Las bajas concentraciones de arsénico en agua presentes en esos sitios, probablemente se deban a que se encuentran cerca de una divisoria de agua subterránea, existiendo dos microcuencas: una que dirige sus flujos hacia el Este y otra hacia el Sur; por lo que la recarga de agua y la interacción del agua-roca presente en cada microcuenca esté confiriendo características químicas diferentes a las aguas, de ahí que en unos pozos se tenga mayor contenido de arsénico y en otros no; a la vez, se debe considerar que la presencia de altas concentraciones de arsénico está asociada a que este acuífero está conformado por un acuífero aluvial somero y un acuífero volcánico por lo que el ascenso de aguas subterráneas profundas enriquecidas con arsénico es inminente a través de las fallas y fracturas, aunado a que esta zona es volcánica y altamente sísmica.

El 30\% de los sitios analizados se encuentran al Este de la comunidad de La Fuente buscando hacia las comunidades de Tecuaname, El Papalonal y Cuatro Palos (Figura 5), y descargan hacia el Río Sinecapa. (Longley A., 2005); mientras que las mayores concentraciones de arsénico en agua se reportan hacia la caldera Galán. Dado las bajas concentraciones encontradas hacia el sector Este de la zona de estudio, se sugiere promover el uso de estas aguas como fuentes seguras de abastecimiento de agua potable para consumo humano, lavar alimentos, lavarse los dientes y cocinar. 


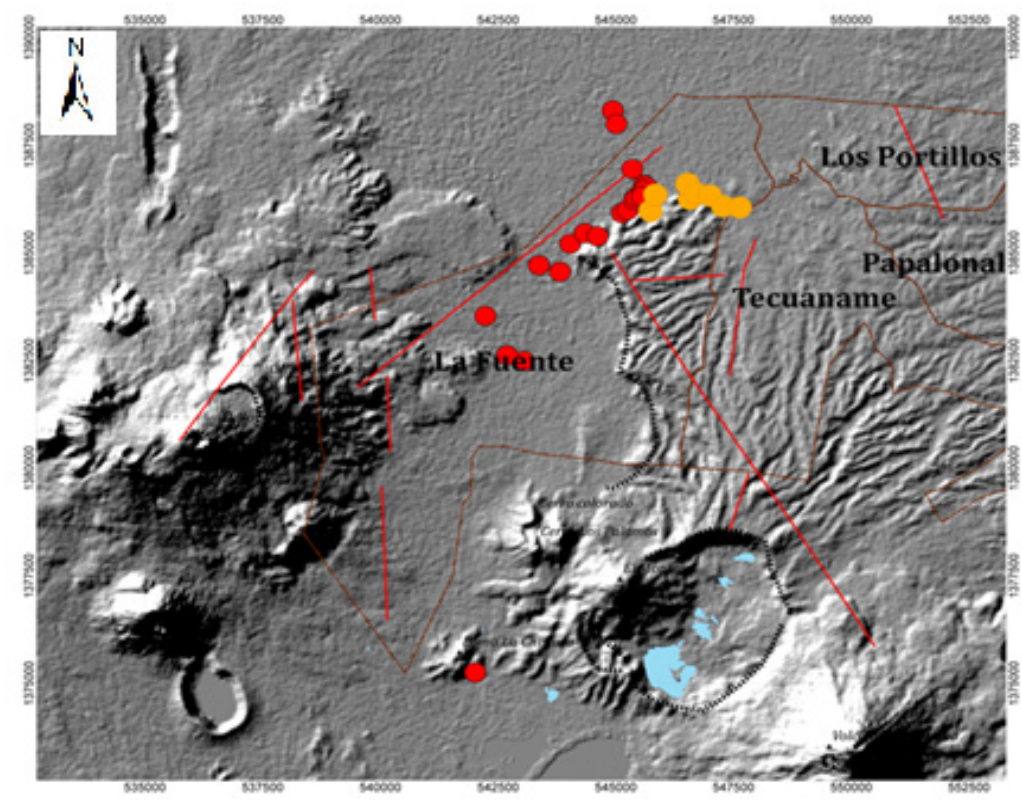

Figura 5. Mapa de concentraciones de arsénico, fallas y calderas volcánicas.

\section{Presentación de resultados ante la comunidad y actores locales}

El 25 de mayo del 2017 se realizó una visita a la comunidad rural La Fuente, llevándose a cabo la presentación del estudio en el colegio Santa Ana de esta comunidad. La exposición se realizó ante: 1) la comunidad de la Fuente en general; 2) las autoridades locales de los CAPs del Terrero, Tecuaname, El Papalonal y La Fuente; 3) el encargado de Gestión de Riesgo de la alcaldía de La Paz Centro, el Cro. César Torres Salgado; 4) los miembros del grupo Samaritan's Purse Canada; y 5) la directora del colegio La Fuente. En la presentación se informó sobre la calidad de las aguas subterráneas de acuerdo a la concentración de arsénico presente en cada una de ellas, así como los daños irreversibles que este metaloide causa en la salud humana; por último, se orientó a la población y autoridades presentes sobre el cambio de uso de las fuentes de agua actuales por las fuentes alternativas y de agua segura para la población, las cuales equivalen al $30 \%$ de los pozos analizados y que cumplen con los valores guías establecidos para agua de consumo humano (10 $\mu \mathrm{g} \cdot \mathrm{l}^{-1}$; OMS, 2011).

Esta investigación, además de generar información científica inédita en esta zona de estudio, se convierte en una herramienta para: 1) apoyar en la toma de decisiones por parte de las autoridades, en cuanto a las fuentes de suministro seguras de agua potable para la población; 2) a que los pobladores locales realicen un cambio en el uso de las fuentes alternativas para agua de consumo humano y de preparación de sus alimentos; 3) para que las entidades de gobierno como alcaldía de La Paz Centro, el Ministerio de Salud (MINSA) y la Autoridad Nacional del Agua (ANA) cuenten con antecedentes para la ejecución de futuros proyectos de agua en lo referente a la calidad de la misma, y que sirva como antecedente para la toma de decisiones en la planificación y ordenamiento territorial del municipio; y 4) para que las autoridades gubernamentales y el 
MINSA realicen estudios de salud a la población enfocándose en la prevalencia de este tóxica en las aguas subterráneas.

\section{CONCLUSIÓN}

La mayoría de los pozos monitoreados en la comunidad de La Fuente superan el valor de concentración total de arsénico admisible $\left(10 \mu \mathrm{g} \cdot \mathrm{l}^{-1}\right)$ en agua para la ingesta humana establecido por la Organización mundial de la Salud y adoptado por el Instituto Nicaragüense de Acueductos y Alcantarillados como norma de calidad para uso nacional.

En el área de estudio el arsénico es de origen geogénico por estar ubicada en la base de las faldas septentrionales del volcán El Hoyo y del edificio de la caldera Galán.

La intensa alteración hidrotermal en esta área, producto del vulcanismo reciente del Cuaternario que propicia la liberación de arsénico desde las estructuras mineralizadas, así como por el ascenso de aguas subterráneas enriquecidas con arsénico a través de fallas y fracturas que sirven como conducto del contaminante.

Las fuentes de agua que cumplen con los límites máximos admisibles para agua de consumo humano (10 $\mu \mathrm{g} \cdot \mathrm{l}^{-1}$ de arsénico) en la comunidad de La Fuente, son recomendadas como fuentes de agua segura para consumo de la población.

Se presume que el sector Este de la zona de estudio, buscando hacia la comunidad de Tecuaname pueden ser los sitios recomendados para perforar nuevos pozos de suministro de agua segura para la población, previos estudios geológicos, hidrogeológicos y de calidad del agua.

La divulgación de los resultados generó el empoderamiento de la población de la comunidad de La Fuente cambiando la cultura del uso del agua, usando las fuentes de agua de buena calidad, usando métodos alternativos de remoción de arsénico (filtros de ósmosis inversa), lo que derivará en la disminución de las patologías relacionadas con este carcinógeno.

\section{BIBLIOGRAFÍA}

Altamirano, M. (2005). Contaminación Natural por arsénico en las aguas subterráneas de la subcuenca sur oeste Valle de Sebaco- Matagalpa.

Armienta , M. A., \& Segovia, N. (2008). Arsenic and fluoride in the groundwater of Mexico.Environmental Geochemistry Health, 30, 345.

Anawar, H. J., Akai, K., Komaki, H., Terao, T., Yosioka, T., Ishizuka, S., et al. (2003). Geochemical occurrence of arsenic in the groundwater of Bangladesh sources and mobilization processes.

ATSDR. (2007). Reseña toxicológica del plomo. Atlanta, EE. UU.

Boyle, R. (1979). The Geochemistry of Gold and its deposits (together with a chapter 
on geochemical prospecting for the element Bulletin.

CAPRE. (1994). Normas de Calidad del Agua para Consumo Humano. Obtenido de ENACAL: http://biblioteca.enacal. com.ni/bibliotec/Libros/pdf/CAPRE_ Normas_Regional.pdf

CIRA/UNAN-Managua, \& Laboratorio Contaminantes Metálicos. (2009). Análisis termales Tipitapa. Managua.

Electroconsult. (Octubre de 2001). Plan Maestro Geotérmico de Nicaragua Evaluación del Área de El Hoyo- Monte Galán. Comisión Nacional de Energía, VI.

Esperanzas, N. (2010b). Estudio de arsénico en Telica. Una investigación de la extensión, las causas y los efectos de la contaminación por arsénico en el Municipio de Telica, Departamento de León. Telica.

Gómez C., A. (2002). Monitoreo y Atención de intoxicados con Arsénico en El Zapote, Municipio de San Isidro, Departamento de Matagalpa, Nicaragua 1994-2002. Managua: UNICEF.

Gómez Cuevas, A. (2007). Efectos cutáneos y respiratorios en personas con intoxicación crónica con arsénico en agua en El Zapote, Municipio de San Isidro - Matagalpa, Nicaragua 2007. Tesis de Maestría, Managua.

Hossain (2006). Alteraciones cardiovasculares, renales, circulatorias y respiratorias, en última instancia.
INAA. (2001). Normas Técnicas para el diseño de abastecimiento y potabilización de agua. Normas NTOM 09003-99. Managua.

Kinniburgh, D G; Smedley, P L; Summary. (2001). Arsenic contamination of groundwater in Bangladesh. BGS Technical Report WC/00/19, British Geological Surve (Vol. 1).

Longley, A. (2005). La Palmerita: Estudio de Factibilidad de Agua.

Martínez, W. (1999). TheInterrelationship between Volcanic and seismic Activity to Subduction - Related tectonics in Western Nicaragua. Jour. Geol.Soc. Japan.

Nuevas Esperanzas. (2013). Annual Report. León.

Nuevas Esperanzas, \& Longley, A. (2010). Estudio Hidrogeológico. Proyecto de Agua Potable La Unión, Nuevo Amanecer y El Cortezal, Municipio de Telica. Informe Final, Telica.

OLADE. (1982). Aprovechamiento de Los Recursos Geotérmicos de la República de Nicaragua. Estudio de Prefactibilidad del Area El Hoyo - San Jacinto: a) Anexo 10, Fichas de los Puntos de Agua Termales, y, c) Anexo 21. Tabulación de Puntos de Muestreo y Datos Hidroquímico.

OLADE;. (1982). Aprovechamiento de Los Recursos Geotérmicos de la República de Nicaragua. Estudio de Prefactibilidad del Area El Hoyo - San Jacinto: a) Anexo 10, Fichas de los Puntos de Agua Termales, 
y, c) Anexo 21. Tabulación de Puntos de Muestreo y Datos Hidroquímico.

OMS. (2006). Organización Panamericana de la Salud Guías para la Calidad del agua potable Vol.2 .

OMS. (2006). Aspectos microbiológicos. Obtenido de Guías para la calidad del agua potable - Vol 1: Recomendaciones: https://www.who.int/water_sanitation_ health/publications/gdwq3/es/

OMS. (2011). Guías para la calidad del agua de consumo humano, Cuarta edición que incorpora la primera ADENDA.

OMS. (2011). Guidelines for drinking Water quality ( 3a edición). World Health Organization, 1.

ORGANIZACIÓN PANAMERICANA DE LA SALUD, OPS/OMS Nicaragua, \& Nuevas Esperanzas. (2011). Estudio de la Contaminación del Agua por Arsénico en el
Municipio de Télica, León. Informe Final, Télica.

Smedley, P., Nicolli, B., Macdonald, A., \& Barros, J. (2002). Hidrogeochemistry of arsenic and other inorganic constituents in groundwaters from La Pampa Argentina.

Viramonte, M. (2001). Mapa geológico del complejo volcánico El Hoyo. Revista geologica America central 247, 79-86.

WHO. (2011). World Health Organization. Guidelines for drinking Water quality (3a edición), vol.1. WHO. 